\section{Faserjahre, Asbestbelastung der Lungen, Asbestosen}

Zusammenfassung: Die Stärke der Beziehungen zwischen den berechneten kumulativen Asbestfaserstaubdosen am Arbeitsplatz und den staubanalytisch ermittelten Asbestbelastungen der Lungen wurde an drei entsprechenden Patientenkollektiven (Gesamtkollektiv $n=366$, Kollektiv ohne erhöhte Asbestbelastung der Lungen $n=193$, Kollektiv mit Minimalasbestosen/Asbestosen $n=46$ ) des Deutschen Mesotheliomregisters geprüft. Generell zeigt sich eine nur geringe Beziehung zwischen den beiden Größen. Der arbeitsmedizinisch definierte Grenzwert von $\geq 25$ Faserjahren wurde für 19,6\% der Versicherten ohne vergleichsweise erhöhte Asbestbelastungen der Lungen ermittelt. $24 \%$ des Gesamtkollektivs zeigen ebenfalls keine oder eine nur gering erhöhte Asbestbelastung der Lungen trotz Erreichens oder Übersteigens der Asbestfeinstaubkonzentrationen von 25 Faserjahren. Demgegenüber wurden bei 42,5\% der Versicherten mit deutlich erhöhten Asbestbelastungen und Ausbildung asbestassoziierter Lungenfibrosen arbeitstechnisch weniger als 25 Faserjahre ermittelt. Diese Datenlage lässt es zweifelhaft erscheinen, den Grenzwert von 25 Faserjahren als geeigneten Parameter für die gutachterliche Beurteilung möglicher asbestassoziierter fibrosierender Lungenveränderungen heranzuziehen.

Fibre-years, asbestos burden of the lungs, and asbestoses: The relations between cumulative asbestos fibre doses at the working places and asbestos burden of the lungs evaluated by lung dust analyses have been tested on three different groups of patients of the German Mesothelioma Registry (1. whole collective $n=366,2$. collective without elevated asbestos burden of the lungs $n=193,3$. collective with minimal asbestoses/asbestoses $n=46$ ). The relations between the above mentioned parameters are in general only weak. The limit value of $>25$ fibre-years (in terms of German industrial medicine) is found in $19.6 \%$ of persons without increased asbestos burden of the lungs. In spite of reaching or even exceeding the cumulative dosis of 25 fibre-years, $24 \%$ of the whole collective also show no or only slightly raised asbestos concentrations in the lung tissues. By contrast, $42.5 \%$ of patients with evidently increased asbestos burden of the lungs and development of asbestos-associated lung fibroses do not attain 25 fibre-years at their working places. Considering our data, it is doubtful whether the postulated limit value of 25 fibre-years can be an adequate parameter for the evaluation of asbestos-associated lung fibroses.

Pneumologie 2000; 54: 155-159

(c) Georg Thieme Verlag Stuttgart · New York

ISSN 0934-8387
M. Fischer, S. Günther, K.-M. Müller

Deutsches Mesotheliomregister Bochum

(Leiter: Prof. Dr. med. K.-M. Müller, Direktor des Institutes für Pathologie) Berufsgenossenschaftliche Kliniken Bergmannsheil Bochum

\section{Einleitung}

Seit 1992 sind Asbestfaserjahre ein wichtiger Bestandteil des Berufskrankheitenrechtes (BK-Ziffer 4104) insofern, als dieser Parameter zur Abschätzung des relativen Lungenkrebsrisikos herangezogen wird. Die Aufnahme der sog. „technischen Brücke“ von 25 Faserjahren (als postulierte Tumorverdopplungsdosis) in den Verordnungstext erfolgte trotz der seinerzeitigen deutlich divergierenden Ergebnisse der mit der Thematik befassten Arbeitsgruppen [1,2,3,4,5,6] und trotz begründeter Einwände von in der Problematik ausgewiesenen Experten [7]. Selbst heute liegen divergierende Berechnungen hinsichtlich des Zusammenhanges zwischen kumulativer Asbestfaserstaubdosis und relativem Risiko asbestassoziierter gutartiger und bösartiger Erkrankungen vor [8,9].

\section{Definition}

Das Faserjahr ist weder eine physikalische Maßeinheit noch eine technische Messgröße, sondern ein vergleichsweise grobes arbeitsmedizinisches Schätzmaß für die Höhe bzw. die Intensität einer Asbestexposition am Arbeitsplatz. Es ist eine abgeleitete Größe und wird definiert als Produkt aus der Konzentration der Asbestfasern länger/gleich $5 \mu \mathrm{m}$ am Arbeitsplatz (Anzahl Fasern $/ \mathrm{cm}^{3}$ bzw. Anzahl Fasern $\times 10^{6} / \mathrm{m}^{3}$ ), der Expositionszeit (als Anteil der Jahresarbeitszeit) und der Gesamtdauer der mit der Exposition verbundenen Tätigkeit oder Tätigkeiten (Jahre).

Sonstige arbeitsplatzspezifische Faktoren wie z.B. Temperatur, Belüftungen mit resultierenden unterschiedlichen Verteilungsmustern oder -geschwindigkeiten freigesetzter Fasern gehen nur bedingt in die Berechnungen mit ein. Vielfach liegen weder genaue Konzentrationsmessungen vor noch sind die Expositionszeiten exakt zu ermitteln [9]. Vor dem Hintergrund häufig fehlender konkreter Messdaten an früheren Arbeitsplätzen wurden Rechenmodelle von einer Kommission des Hauptverbandes der Gewerblichen Berufsgenossenschaften erarbeitet, die im Faserjahr-Report [10] zusammengefasst sind. In einer nicht unerheblichen Zahl der Fälle geht der zuständige Ermittler von den angenommen schlechtesten Bedingungen aus und führt sog. worst-case-Analysen durch, die jedoch mit den realen Verhältnissen gar nicht oder nur entfernt übereinstimmen müssen.

Auch wird der Tatsache, dass rechnerisch identische kumulative Asbestfaserstaubdosen auf durchaus unterschiedlichen Expositionsbedingungen beruhen können, keine Beachtung 
geschenkt. So wird bei z.B. einer kumulativen Dosis von 25 Faserjahren nicht unterschieden, ob sie durch eine Exposition von $25 \times 10^{6}$ Fasern $/ \mathrm{m}^{3}$ über die Dauer eines Jahres oder eine Exposition von $1 \times 10^{6}$ Fasern/m $\mathrm{m}^{3}$ über die Dauer von 25 Jahren zustande kam.

\section{Asbestbelastung der Lungen}

Die Konzentration der im Lungengewebe abgelagerten Asbestfasern ist das Resultat folgender Einflussgrößen:

- Intensität der Asbestexposition und ggf. Intervalldauer

- Fasergeometrie und Faserart

- Biobeständigkeit der Fasern

- Verhältnis von Inhalations-/Eliminations-/Retentionsverhalten des betroffenen Organismus in Abhängigkeit von den individuellen Gegebenheiten.

Die letztgenannten Größen werden maßgeblich durch die jeweiligen Situationen im Lungen- und Bronchialgewebe wie z.B. vorbestehende Gewebeschädigungen bestimmt. Ebenso sind individuelle Verhaltensweisen der Versicherten wie Rauchgewohnheiten, Arbeitstempo und Höhe der Atemfrequenz und des Atemvolumens wichtige Einflussfaktoren.

Die aktuelle Asbestbelastung der Lungen wird durch staubanalytische Untersuchungen quantifiziert. Die lichtmikroskopische Methode erfasst die vom Organismus umhüllten Asbestfasern, die Asbestkörper. In der elektronenmikroskopischen Analyse werden die nicht umhüllten Asbestfasern bestimmt. Naturgemäß sind die durch die elektronenmikroskopischen Untersuchungen ermittelten Faserkonzentrationen um ein Vielfaches höher als die in der lichtmikroskopischen Staubanalytik erhaltenen Anzahlen der Asbestkörper. Die Ergebnisse beider Methoden lassen aber - unter Bezug auf die für sog. Normallungen erhobenen Konzentrationsdaten - Aussagen über Erhöhung oder Nicht-Erhöhung der Asbestbelastung des Lungengewebes zu.

\section{Material und Methode}

Die in verschiedenen Studien bei der Erarbeitung des möglichen Zusammenhanges zwischen den berechneten kumulativen Asbestfaserstaubdosen und dem relativen Erkrankungsrisiko nachgewiesenen Abweichungen legen es nahe, an eigenen entsprechenden Kollektiven die Stärke der Beziehungen der diskutierten Größen zu prüfen.

Die für diese Untersuchungen herangezogenen Daten des Deutschen Mesotheliomregisters werden zunächst ohne Berücksichtigung der Grunderkrankungen der jeweiligen Versicherten analysiert, da die unterschiedlichen Krankheitsbilder bei der Erörterung der anstehenden Problematik eine untergeordnete Rolle spielen.

Analysiert wurde ein Kollektiv von 366 Patienten (Alter zwischen 33 und 82 Jahren), für die sowohl Berechnungen der kumulativen Asbestfaserstaubdosen am Arbeitsplatz (mit Werten zwischen 0,094 bis 314 Faserjahren) vorlagen als auch die Ergebnisse lungenstaubanalytischer Untersuchungen (mit Asbestkörperkonzentrationen von $2 / \mathrm{cm}^{3}$ bis zu $1510400 / \mathrm{cm}^{3}$ ) zur Verfügung standen.
Die Beziehung zwischen dem arbeitsmedizinisch definierten Grenzwert von 25 Faserjahren und den Asbestbelastungen der Lungen wurde gezielt an zwei Unterkollektiven näher getestet:

n = 193 Patienten ohne staubanalytisch erhöhte Asbestbelastung der Lungen

$\mathrm{n}=46$ Patienten mit deutlich erhöhter Asbestbelastung der Lungen und asbestassoziierten fibrosierenden Lungenveränderungen (78\% pathologisch-anatomisch gesicherte Minimalasbestosen, $22 \%$ Asbestosen).

Als Vergleichskollektiv wurden die Daten von $n=46$ Patienten mit malignen Mesotheliomen und elektronenmikroskopisch ermittelten Faserkonzentrationen untersucht. Die Daten entstammen dem Anhang der Studie „Risiko- und Einflussfaktoren des diffusen malignen Mesothelioms (DMM)“ [11].

\section{Ergebnisse}

Zunächst könnte man davon ausgehen, dass die vom Technischen Aufsichtsdienst berechneten unterschiedlich stark erhöhten Asbestfaserkonzentrationen am Arbeitsplatz zwangsläufig zu entsprechend erhöhten Asbestfaser- oder körperkonzentrationen im Lungengewebe führen und im Idealfall eine lineare Abhängigkeit zwischen den beiden Größen zu postulieren ist.

Lineare oder nahezu lineare Abhängigkeiten existieren zwar grundsätzlich in biologischen Systemen, allerdings i.d.R. in nur sehr eng gefassten Bereichen. So ist eine lineare Relation zwischen den hier diskutierten Größen für sehr hohe Asbestfaserstaubdosen vorstellbar, in der Mehrzahl der Untersuchungen liegen derartige Extrembedingungen jedoch nicht vor.

Betrachtet man die Asbestkörperkonzentrationen in den Lungen von 366 Versicherten in Abhängigkeit von den für diese Personen ermittelten kumulativen Asbestfaserstaubdosen (Faserjahre) am Arbeitsplatz, so zeigt das Scatterdiagramm zunächst eine Verteilung der staubanalytischen erhobenen Daten parallel zur X-Achse (Abb.1). Für identische Höhen der berechneten kumulativen Asbestfaserstaubdosen werden also sehr niedrige bis deutlich erhöhte Asbestbelastungen der Lungen nachgewiesen. Darüber hinaus sind nennenswerte Unterschiede in der Verteilung der Daten oberhalb und unterhalb der Linie 25 Faserjahre ebenfalls nicht zu verzeichnen.

Die arbeitstechnischen Ermittlungen für 193 Versicherte ohne staubanalytisch erhöhte Asbestbelastung der Lungen ergaben in 19,6\% der Untersuchungen eine kumulative Asbestfaserstaubdosis von 25 Faserjahren und mehr (Abb. 2).

Auch die Relationen zwischen den elektronenmikroskopisch ermittelten Asbestfaserkonzentrationen der kritischen Abmessungen und den geometrischen Asbestfaserstaubdosen (Faserjahre) von 46 Patienten mit malignen Mesotheliomen zeigen eine breite Streuung der Datenpaare (Abb. 3).

Staubanalytisch finden sich in allen 46 Fällen mit asbestassoziierten Lungenveränderungen auch erhöhte Asbestbelastungen der Lungen. Der Wert von 25 Faserjahren und mehr wird 

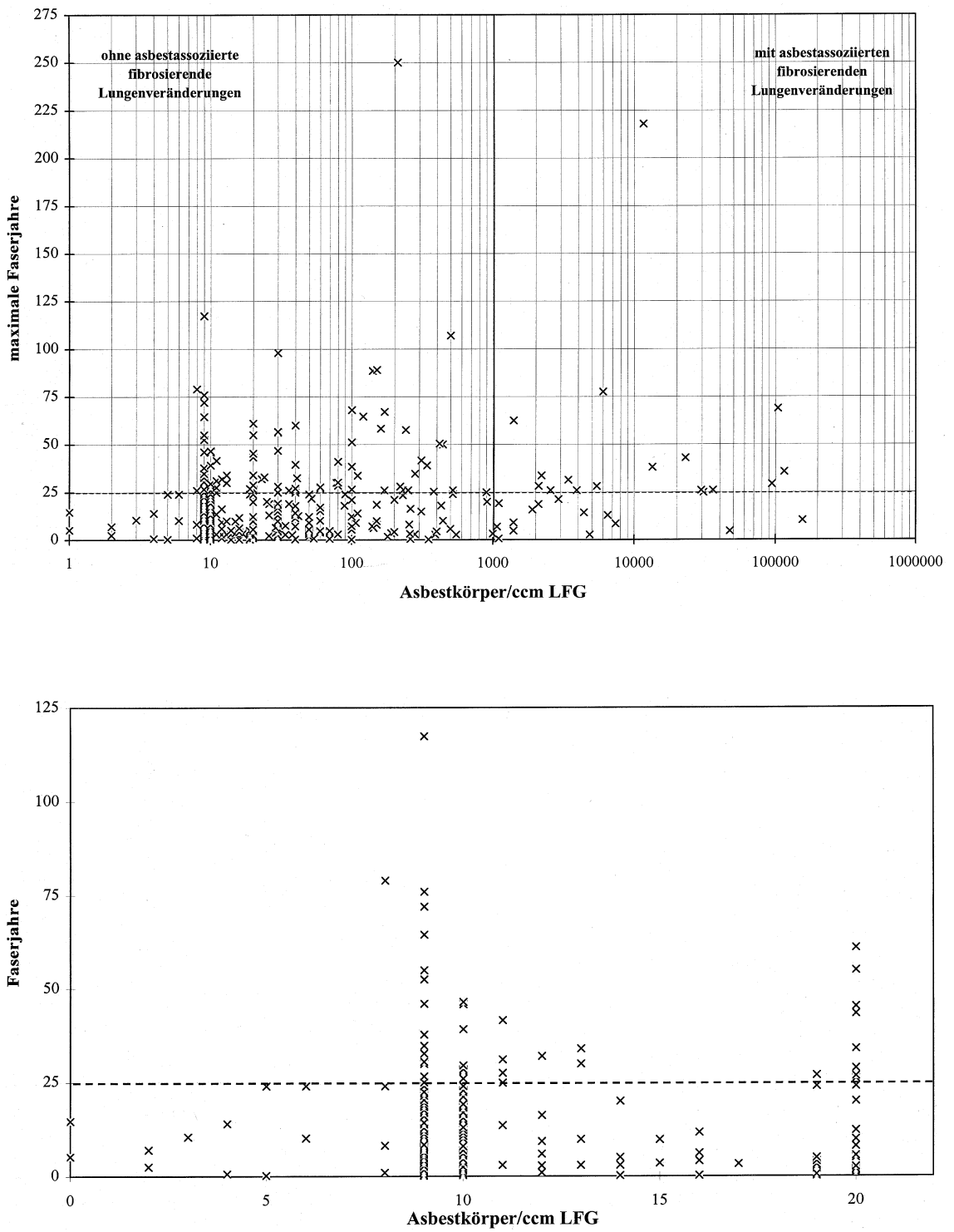

Abb. 1 Relationen zwischen den arbeitstechnisch ermittelten Asbestfaserstaubdosen (Faserjahre) und den staubanalytisch nachgewiesenen Konzentrationen der Asbestkörper im Lungengewebe ( $n=366$ Patienten).
Abb. 2 Kumulative Asbestfaserstaubdosen (Faserjahre) bei $n=193$ Patienten ohne staubanalytisch erhöhte Asbestbelastung der Lungen (bis 20 Asbestkörper/ $\mathrm{cm}^{3}$ Lungengewebe). aber nur für 57,5\% der Versicherten angegeben (Abb. 4). Bei ausschließlicher Anwendung des Kriteriums der 25 Faserjahre wäre also bei $42,5 \%$ der Versicherten die Diagnose einer Minimalasbestose oder Asbestose gar nicht erst in Betracht gekommen.

Im Gegensatz dazu sind bei 24\% (89 von 366) der Versicherten Faserjahrwerte ermittelt, die teilweise deutlich über 25 liegen, bei denen keine oder nur gering erhöhte Asbestbelastungen der Lungen nachgewiesen sind und die - selbst bei Vorliegen von Lungenfibrosen - sicher keine Minimalasbestosen oder Asbestosen entwickelt haben (Abb.1).

\section{Diskussion}

\section{Generelle Aspekte}

Die Verteilungsmuster der Daten (Abb.1-3) lassen nur eine Interpretation zu: Eine strikte Abhängigkeit zwischen der Höhe der kumulativen Asbestfaserstaubdosis und der Höhe der Asbestbelastung der Lungen besteht nicht. Das wiederum bedeutet, entgegen anderslautenden Rechenmodellen, dass aus der Anzahl der errechneten Faserjahre im Einzelfall nicht auf die Höhe der Konzentrationen der im Lungengewebe abgelagerten Asbestkörper bzw. Asbestfasern rückgeschlossen werden kann und umgekehrt.

Die nur geringen Beziehungsstärken der beiden Parameter Asbestfaserjahre und Asbestbelastung der Lungen sind zum einen sicher direkte Folge der Ungenauigkeiten der Faserjahrberechnungen selbst [12].

Aber auch aus patho-physiologischer und pathogenetischer Sicht überrascht dieses Ergebnis nicht. Es ist für den Organismus eben doch von entscheidender Bedeutung, ob z.B. eine Auseinandersetzung über vergleichsweise kurze Zeiträume mit hohen Faserkonzentrationen erfolgen muss oder ob mehrjährige Reaktionen der Lunge auf niedrige Faserdosen ablaufen können [13]. Es ist daher unverständlich, dass auf unterschiedlichen Expositionsbedingungen beruhende, rechnerisch 


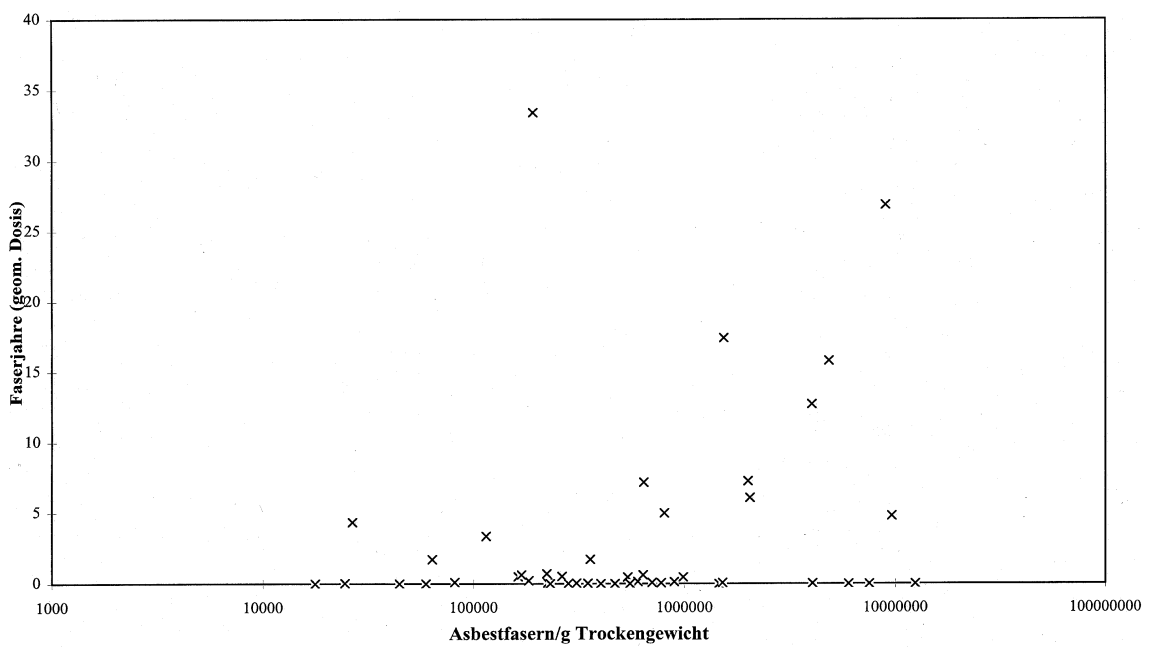

Abb. 3 Kumulative Asbestfaserstaubdosen (Faserjahre) und Konzentrationen von Asbestfasern der kritischen Abmessungen (Längen $\geq 5 \mu \mathrm{m}$ ) in Lungengeweben von $\mathrm{n}=46$ Patienten mit malignen Mesotheliomen. Aus: Risiko- und Einflussfaktoren des diffusen malignen Mesothelioms, H.-J. Woitowitz et al., Anhang 10.2, 1993.

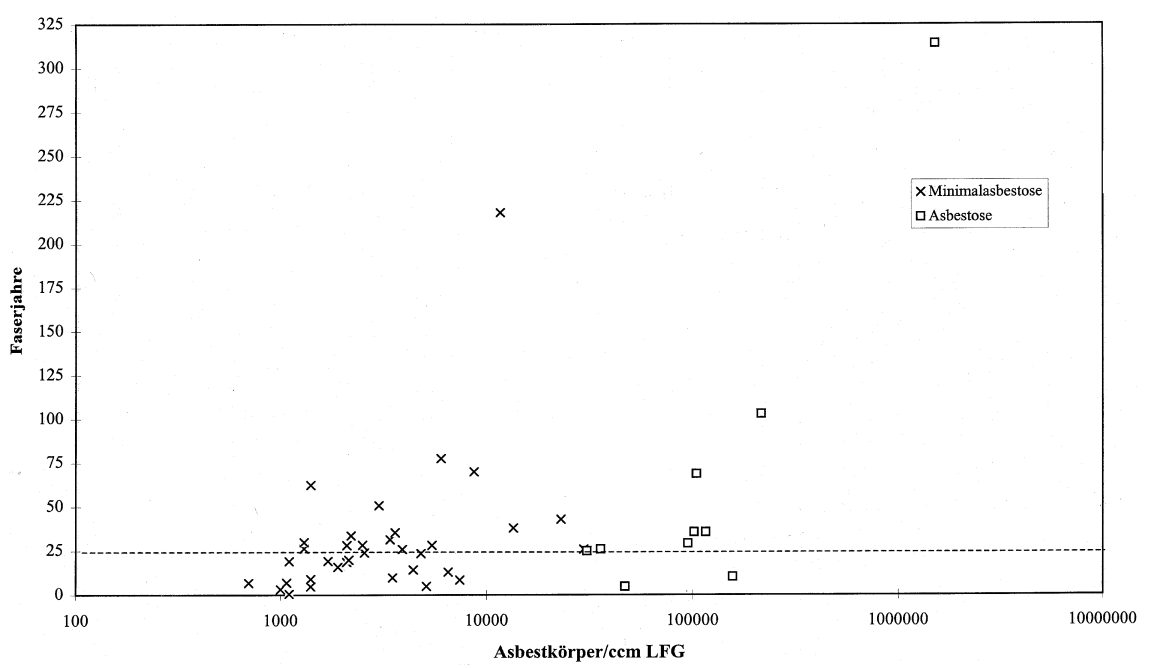

Abb. 4 Beziehungen zwischen den kumulativen Asbestfaserstaubdosen, den Asbestbelastungen der Lungen und asbestassoziierten fibrosierenden Lungenveränderungen (Minimalasbestosen/Asbestosen) bei $\mathrm{n}=46$ Patienten

aber identische Asbestfaserstaubdosen in ihrer Wirkung auf den Organismus in undifferenzierter Weise gleichgesetzt werden. Auch das oben beispielhaft angeführte Spektrum der individuellen Faktoren spielt eine bedeutende Rolle für die möglichen Auswirkungen einer erhöhten Faserexposition und ist in dem Faserjahrmodell in u.E. unvertretbarer Weise vernachlässigt.

\section{Asbestfeinstaubkonzentrationen (Faserjahre) und Asbestosen}

Die Asbestfaserstaubkonzentration von 25 Faserjahren wird, nach epidemiologisch definierten Kriterien, nicht nur als Verdopplungsdosis des Lungenkrebsrisikos angesehen, sondern in letzter Zeit immer intensiver auch als arbeitsmedizinischer Grenzwert für die Entwicklung bzw. Ableitung asbestassoziierter fibrosierender Lungenveränderungen im Sinne einer Minimalasbestose oder Asbestose diskutiert. Der Grund für diese unter histomorphologischen Aspekten und unter Berücksichtigung der Helsinki-Kriterien schwer nachvollziehbare Diskussion ist wohl darin zu sehen, dass zunächst die minimalen asbestbedingten Fibrosierungen im Lungengewebe der Analyse durch bildgebende Verfahren entzogen und allein pathologisch-anatomisch verifizierbar sind. Im gegen- läufigen Sinne ist das Bestreben offenkundig, radiologisch oder computertomographisch relativ häufig darstellbare unspezifische fibrosierende Lungenveränderungen - bei arbeitstechnisch entsprechend gesicherter Asbestexposition - als Asbestosen zu werten. Dies allerdings ohne die histomorphologische Kontrolle durch den Pathologen und unter Missachtung der Tatsache, dass fibrosierende Lungenprozesse ganz unterschiedlicher kausaler und formaler Pathogenese ähnliche bzw. sogar vergleichbare Strukturveränderungen in den bildgebenden Verfahren zeigen.

Die hier erhobenen Daten (Abb.1 und 4) machen deutlich, dass die hohen Prozentsätze falsch-negativer (42,5\%) und möglicher falsch-positiver Bewertungen (24\%) nicht erkennen lassen, dass das Kriterium der 25 Faserjahre die für einen Beurteilungsparameter in gutachterlichen Fragestellungen asbestassoziierter Lungenfibrosen $\mathrm{zu}$ fordernde Zuverlässigkeit erfüllt.

Wir halten es daher für überdenkenswert, den Parameter der Asbestfaserjahre als alleiniges Beurteilungskriterium für die Begutachtung asbestassoziierter fibrosierender Lungenveränderungen heranzuziehen. Wir verkennen dabei nicht das 
durchaus legitime Bemühen um Definitionen von Äquivalenzdosen und Grenzwerten [14,15,16], um für möglichst viele Versicherte die geforderte Wahrscheinlichkeit der Zusammenhänge von Erkrankungen und beruflichen Tätigkeiten zu ermöglichen. Dieses sicher auch vor dem Hintergrund des Bestrebens, die gesetzliche Basis für die Definition neuer Berufskrankheiten eher in dem Bereich sozialpolitischer Entscheidungen anzusiedeln: „Wir müssen uns selbstverständlich darüber im klaren sein, dass die BK-rechtliche Umsetzung solcher Erkenntnisse über multifaktoriell verursachte Krebserkrankungen nicht eine wissenschaftsbasierte, also allein wissenschaftliche Entscheidung ist, sondern weitgehend eine sozialpolitische Wertentscheidung, für die die Wissenschaft nicht verantwortlich gemacht werden kann und soll“ [17]. Ungeachtet der Tatsache, dass die gesetzliche Entscheidungsgrundlage auch heute über die Erkenntnisse der medizinischen Wissenschaft definiert ist, möchten wir bei der vorgestellten eindeutigen Datenlage mit der Möglichkeit einer vergleichsweise hohen Anzahl sowohl falschnegativer als auch falschpositiver Befundungen unserer Sorge darüber Ausdruck verleihen, dass es im Rahmen von Berufskrankheitenverfahren zu sozial ungerechten Entscheidungen kommen kann.

\section{Literatur}

${ }^{1}$ Enterline P, De Coufle P, Henderson V. Respiratory cancer relation to occupational exposures among retired asbestos workers. $\mathrm{Br} \mathrm{J}$ Ind Med 1973; 30: 162 - 166

${ }^{2}$ Selikoff IJ. Cancer risk of asbestos exposure. In: Origins of Human Cancer. 1977

${ }^{3}$ Peto J. The hygiene standard for chrysotile asbestos. The Lancet 1978; 4: $484-489$

${ }^{4}$ Mc Donald JC, Liddell FDK, Gibbs GW, Eyssen GE, Mc Donald AD. Dust exposure and mortality in chrysotile mining, 1910-1975. Br J Ind Med 1980; 37: 11 - 24

${ }^{5}$ Dement JM. Estimation of dose and evaluation of dose-response in a retrospective cohort mortality study of chrysotile asbestos textile workers. Doctoral Dissertation, University of North Carolina, School of Public Health, Chapel Hill. North Carolina: 1980

${ }^{6}$ Dement JM, Harris RL, Symons MJ, Shy C. Estimates of DoseResponse for Respiratory Cancer among Chrysotile Asbestos Textile Workers. Ann occup Hyg 1982; 20, 1 - 4: 869 - 887

${ }^{7}$ Lehnert G, Raithel HJ, Valentin H. Asbestfeinstaubexposition, Asbestose und Lungenkrebs. ASP 1992; 27,3: 96 - 101

${ }^{8}$ Lash TL, Crouch EA, Green LC. A meta-analysis of the relation between cumulative exposure to asbestos and relative risk of lung cancer. Occup Environ Med 1997; 54,4: 254 - 263

${ }^{9}$ Burdorf A, Swuste P. An Expert System for the Evaluation of Historical Asbestos Exposure as Diagnostic Criterion in Asbestos-related Diseases. Ann occup Hyg 1999; 43,1: 57 - 66

10 BK-Report 1/97. „Faserjahre“ Hauptverband der Gewerblichen Berufsgenossenschaften. 3. Auflage, 1996. 1 - 200

${ }^{11}$ Woitowitz H-J, Hillerdal G, Calavrezos A, Berghäuser KH, Rödelsperger K, Jöckel K-H. Risiko- und Einflussfaktoren des diffusen malignen Mesothelioms (DMM). Schriftenreihe der Bundesanstalt für Arbeitsschutz 1993; FB 698: 1 - 298

${ }^{12}$ Nicholson WJ, Perkel G, Selikoff IJ. Occupational Exposure to Asbestos: Population at Risk and Projected Mortality 19802030. Am J Ind Med 1982; 3: 259 - 311

${ }^{13}$ Peto J. Dose and time relationships for lung cancer and mesothelioma in relation to smoking and asbestos exposure. bga-Schriften, Hrsg: Fischer und Meyer. München: Medizin Verlag, 1984; 2: $126-132$
${ }^{14}$ Rödelsperger K, Römer W, Bödeker R, Brückel B, Arhelger R, Gosch V, Woitowitz H-J. Asbestfaserstaubdosis am Arbeitsplatz und Asbestfaserkonzentrationen in der Lunge. Dtsch Ges Arbeitsmed, 33 Jahrestagung, Wiesbaden. Stuttgart: Gentner, 1993. $117-123$

${ }^{15}$ Rödelsperger K. Anorganische Fasern im menschlichen Lungengewebe. Schriftenreihe der Bundesanstalt für Arbeitsmedizin 1996; Fb 01 HK 076: 1 - 366

${ }^{16}$ Rödelsperger K, Mandi A, Brückel B, Barbisan P, Woitowitz H-J. Anorganische Fasern im Lungengewebe ungarischer und deutscher Lungenkrebspatienten. (in press).

${ }^{17}$ Woitowitz H-J. Stellungnahme bei der Podiumsdiskussion im Fachkolloquium „Berufsgenossenschaftliche Aktivitäten und Forschungsvorhaben zum Thema Wismut-Erkenntnisse und Perspektiven“ 9./10.12.1996 zitiert in: Mehrtens, G und St Brandenburg: Neue Berufskrankheiten auf gesetzlicher Basis oder nach sozialpolitischen Wertentscheidungen? Arbeitsmed Sozialmed Umweltmed 1999; 34,9: 378 - 379

\section{Dr. rer. nat. M. Fischer}

Deutsches Mesotheliomregister Berufsgenossenschaftliche Kliniken Bergmannsheil Bürkle-de-la-Camp-Platz 1 44789 Bochum 